\title{
Oculomotor prediction of accelerative target motion during occlusion: long-term and short-term effects
}

\author{
Simon J. Bennett • Jean-Jacques Orban de Xivry • \\ Philippe Lefèvre · Graham R. Barnes
}

Received: 25 November 2009 / Accepted: 21 May 2010

(C) Springer-Verlag 2010

\begin{abstract}
The present study examined the influence of long-term (i.e., between-trial) and short-term (i.e., withintrial) predictive mechanisms on ocular pursuit during transient occlusion. To this end, we compared ocular pursuit of accelerative and decelerative target motion in trials that were presented in random or blocked-order. Catch trials in which target acceleration was unexpectedly modified were randomly interleaved in blocked-order trials. Irrespective of trial order, eye velocity decayed following target occlusion and then recovered towards the different levels of target velocity at reappearance. However, the recovery was better scaled in blocked-order trials than random-order trials. In blocked-order trials only, the reduced gain of smooth pursuit during occlusion was compensated by a change in saccade amplitude and resulted in total eye displacement (TED) that was well matched to target displacement. Subsidiary analysis indicated that three repeats of blockedorder trials was sufficient for participants to modify eye
\end{abstract}

\author{
S. J. Bennett $(\square)$ \\ Research Institute for Exercise and Sport Sciences, \\ Liverpool John Moores University, Liverpool L3 3AF, UK \\ e-mail: s.j.bennett@ljmu.ac.uk

\section{G. R. Barnes} \\ Faculty of Life Sciences, University of Manchester, \\ Manchester M60 1QD, UK \\ J.-J. Orban de Xivry $\cdot$ P. Lefèvre \\ CESAME, Université Catholique de Louvain, \\ 1348 Louvain-la-Neuve, Belgium \\ J.-J. Orban de Xivry · P. Lefèvre \\ Laboratory of Neurophysiology, \\ Université Catholique de Louvain, \\ 1200 Brussels, Belgium
}

displacement compared to that exhibited in random-order trials, although more trials were required before end-occlusion eye velocity was better scaled. Finally, we found that participants exhibited evidence of a scaled response to an unexpected change in target acceleration (i.e., catch trials), although there were also transfer effects from the preceding blocked-order trials. These findings are consistent with the suggestion that on-the-fly prediction (short-term effect) is combined with memorised information from previous trials (long-term effect) to generate a persistent and veridical prediction of occluded target motion.

Keywords Smooth pursuit · Saccades $\cdot$ Prediction . Occlusion $\cdot$ Acceleration

\section{Introduction}

It has long been recognised that the oculomotor system must predict upcoming target motion in order to minimise retinal slip and/or position error that would result if pursuit were based on negative feedback alone (Bahill and McDonald 1983; Dallos and Jones 1963). However, pursuit would be limited if it were a simple perpetuation of the current state of the stimulus alone (for a review see Barnes 2008). Targets moving in our natural surrounds are subjected to the physical constraints of gravity and friction and hence, do not often have constant velocity; it is well accepted that acceleration can be perceptually discriminated when the resulting change in velocity is above 20 25\% (Babler and Dannemiller 1993; Brouwer et al. 2002; Werkhoven et al. 1992). In addition, moving targets are frequently occluded from view by other objects and will often reappear moving at a different velocity. Consequently, to maintain accurate pursuit, it is necessary for the 
oculomotor system to form a prediction that reflects the non-uniform nature of target motion, and then retain this prediction in the absence of visual feedback, thereby providing a veridical velocity and positional reference for future smooth and saccadic eye movements; for neural correlates of an internal model of occluded visual motion see Barborica and Ferrera (2003), Cerminara et al. (2009), Nagel et al. (2006).

Using an occlusion paradigm in which the target is expected to reappear, it has been shown that the availability of visual feedback prior to occlusion (for duration of at least $500 \mathrm{~ms}$ ) is sufficient to enable participants to scale their ocular response such that it reflects the end-occlusion trajectory (Bennett et al. 2007). Importantly, by presenting trials in random-order and minimising predictive cues from target position and velocity, the above study indicated that participants were able to extract information on accelerative motion, and then use this to form a dynamic internal representation for predicting the occluded trajectory (for additional details see Orban de Xivry et al. 2008). It was also noted that in trials where there was a strong positive acceleration (i.e., $8 \mathrm{deg} / \mathrm{s}^{2}$ ), the predictive response did not entirely eliminate position and velocity error at the moment the target reappeared. This error was not the result of limitations in the maximum positional change and magnitude of velocity that can be produced during ocular pursuit (Leigh and Zee 1991). Furthermore, while participants attempted to offset the developing displacement error using saccades, this only had limited success.

Better correspondence between pursuit eye movements and occluded target motion has been found when the duration of the occlusion and end-occlusion velocity is known in advance. When trials are presented in blocked-order, participants exhibit an anticipatory recovery in eye velocity that matches well an expected step change in target velocity at the moment of reappearance (Bennett and Barnes 2004, Orban de Xivry et al. 2006). Moreover, even though eye displacement resulting from smooth pursuit during occlusion is still somewhat less than target displacement, any discrepancy is minimised by a coordinated response from the saccadic system. It has also been found with constant velocity targets and only in the presence of a reinforcing auditory stimulus that there is a marked increase in smooth pursuit gain during occlusion and a parallel reduction in the frequency of saccades after completing an extensive number of trials (Madelain and Krauzlis 2003). The finding of improved prediction in the oculomotor response during occlusion following repeated attempts is explicable by adaptation of the internal drive (Bennett and Barnes 2006b; Churchland et al. 2003; Madelain and Krauzlis 2003), in combination with increased expectation from previous trials regarding end-occlusion target velocity and/or position
(Becker and Fuchs 1985). These means of improving correspondence between the oculomotor response and target motion have been referred to as long-term forms of prediction (Deno et al. 1995).

To date, it remains unknown whether repeated attempts to pursue accelerative target motion would similarly enable participants to develop a better prediction of non-uniform occluded target motion. Unlike the studies described above where there was a step change in target velocity at reappearance that was not a direct extrapolation of target velocity prior to occlusion, accelerative motion provides endogenous information about the future target trajectory. Therefore, in the typical situation where there would be no auditory reinforcing stimulus, participants could benefit from receiving trials in blocked-order and improve the correspondence between pursuit eye movements and accelerative target motion. In this respect, it is also relevant to question how any modification to the oculomotor response would be reflected in the collaboration between smooth and saccadic eye movements. It is possible that repeated attempts to pursue accelerative target motion might enable participants to better maintain smooth pursuit throughout occlusion and hence reduce the contribution from saccades. Alternatively, there may be no change in smooth pursuit during occlusion but the developing position error could be offset by an increased contribution from saccades (Orban de Xivry et al. 2006, 2008). In the latter case, it would be implied that while participants are unable to modify the internal drive, they are still able to use information from prior trials regarding the target trajectory to control eye displacement.

The present study was designed to answer the above questions, and in doing so extend understanding regarding the influence of both long-term (i.e., between-trial) and short-term (i.e., within-trial) predictive mechanisms that operate in ocular pursuit during transient occlusion. Specifically, we examined ocular pursuit of accelerative and decelerative target motion in trials received in random and then blocked-order. Occlusion duration was known in advance but in random-order trials, target velocity at the moment of occlusion did not provide reliable cues regarding the unseen trajectory, unless acceleration could be perceived. During the blocked-order trials, we also interleaved catch trials in which target acceleration was unexpectedly altered. This provided the opportunity to determine whether participants' prediction of the target motion during occlusion was modified on a trialby-trial basis following an unexpected change in visual feedback from the initial part of the trial. Furthermore, the inclusion of catch trials made it possible to determine if there also were transfer effects from the preceding blocked-order trials compared to like trials received in random-order. 


\section{Methods}

\section{Participants}

Six healthy human participants (mean age: 27 years), all of whom had previous experience of ocular pursuit tasks, took part after giving informed consent. Two of the participants were authors (P1-SJB; P4-JJO), and the others were naïve to the purpose of the study. Participants were, however, told that they would be required to track a moving target that could undergo a transient occlusion. All participants had normal or corrected-to-normal vision were healthy and without any known oculomotor abnormalities. All procedures were conducted with approval of the Université Catholique de Louvain ethics committee.

\section{Apparatus}

Participants sat in a purpose-built dark room, facing a flat white screen $(2 \times 1.5 \mathrm{~m})$ at a viewing distance of $1.5 \mathrm{~m}$. The head was supported with a chin rest that was adjusted to each participant's height. Visual stimuli were projected onto the screen using a CRT projector (Barco Cine8) with a refresh rate of $100 \mathrm{~Hz}$ and $800 \times 600$ spatial resolution. The horizontal motion of the visual stimuli was controlled by a visual stimulus generator (VSG2-5; Cambridge Research Systems Ltd) interfaced with a PC running proprietary software through MATLAB (Mathworks Inc). Eye movements were recorded at $200 \mathrm{~Hz}$ using video-oculography (Chronos Vision C-ETD) and stored to a PC for off-line analysis.

\section{Procedures}

Having been familiarised with the experimental set-up, participants completed 5 sessions, each commencing with a calibration procedure (see Orban de Xivry et al. 2006) followed by sets of trials in test or control conditions. In test conditions (with occlusion), participants completed 32 sets of 30 trials $(n=960)$ arranged in a random-order, followed by 18 sets of 40 trials $(n=720)$ arranged in a blockedorder. Control conditions (without occlusion) in which participants completed 8 sets of 30 trials $(n=240)$ arranged in a random-order were introduced between sets of the random-order test conditions. Participants were informed of the impending condition. Data collection took place over several days and produced a large data set from which a small sub-set was compared for the purposes of the current study.

All trials began with a green central fixation point presented for a period varying randomly between 500 and $1,500 \mathrm{~ms}$. After the fixation period, the green target was replaced by a co-located red target that started to move with constant acceleration either leftward or rightward. In pseudo-randomised, test trials the target moved for 200 , 500 or $800 \mathrm{~ms}$, after which it disappeared behind an imaginary occluder for $800 \mathrm{~ms}$. Initial target velocity was chosen such that target velocity $50 \mathrm{~ms}$ before occlusion (pre-occlusion velocity) was either 0 or $8 \mathrm{deg} / \mathrm{s}$. Target acceleration was constant during the entire trial, hence when the target reappeared for $400 \mathrm{~ms}$ after the occlusion its motion characteristics were a straightforward extrapolation of the previous trajectory. For pre-occlusion target velocity of $8 \mathrm{deg} / \mathrm{s}$, target acceleration was $-8,-4,0,4$ or $8 \mathrm{deg} / \mathrm{s}^{2}$ (see Fig. 1 for representation of target motion characteristics in trials with pre-occlusion target velocity of $8 \mathrm{deg} / \mathrm{s}$ ). For preocclusion target velocity of $0 \mathrm{deg} / \mathrm{s}$, target acceleration was $0,-4,-8,-12$ or $-16 \mathrm{deg} / \mathrm{s}^{2}$. So, when the target initially moved rightwards, it decelerated and reached $0 \mathrm{deg} / \mathrm{s}$ at $50 \mathrm{~ms}$ before occlusion, after which velocity reversed and it moved leftwards throughout the rest of the trial. As a consequence of these velocity and acceleration characteristics, the target reappeared to the left (or right in trials where the initial motion was leftwards) of its disappearance position. The same target motion characteristics were used in control trials but the target remained visible throughout.

In blocked-order test trials the target also moved for $800 \mathrm{~ms}$, after which it disappeared behind an imaginary occluder for $800 \mathrm{~ms}$ and then reappeared for a further $400 \mathrm{~ms}$. Initial target velocity was chosen such that preocclusion velocity was $8 \mathrm{deg} / \mathrm{s}$ and target acceleration in the majority of trials was constant at $-8,0$, or $8 \mathrm{deg} / \mathrm{s}^{2}$. In a minority (i.e., 15\%) of blocked-order trials, catch trials were introduced in which target acceleration was unexpectedly modified by $\pm 4 \mathrm{deg} / \mathrm{s}^{2}$ of the base target acceleration (i.e., -8 to -4 or $-12 \mathrm{deg} / \mathrm{s}^{2} ; 0$ to -4 or $4 \mathrm{deg} / \mathrm{s}^{2} ; 8$ to 4 or $12 \mathrm{deg} / \mathrm{s}^{2}$ ). The catch trials were pseudo-randomly interleaved between 3 and 12 repeats of the base target acceleration. A low percentage of catch trials was used in order to minimise the influence on behaviour in the surrounding blocked-order trials. Participants were instructed to track the target as accurately as possible throughout the presentation.

\section{Data extraction}

Eye position signals were processed using procedures adopted from de Brouwer et al. (2002) and first involved low-pass filtering at $50 \mathrm{~Hz}$ using an autoregressive, zerophase digital filter implemented in MATLAB (Mathworks, Inc). Eye velocity and acceleration were then derived from filtered position signals using a weighted central difference algorithm on a $\pm 10 \mathrm{~ms}$ time interval. Saccades were detected using a $500 \mathrm{deg} / \mathrm{s}^{2}$ acceleration threshold and were considered to occur during the occlusion when their onset was at least $100 \mathrm{~ms}$ after the start of the occlusion and 

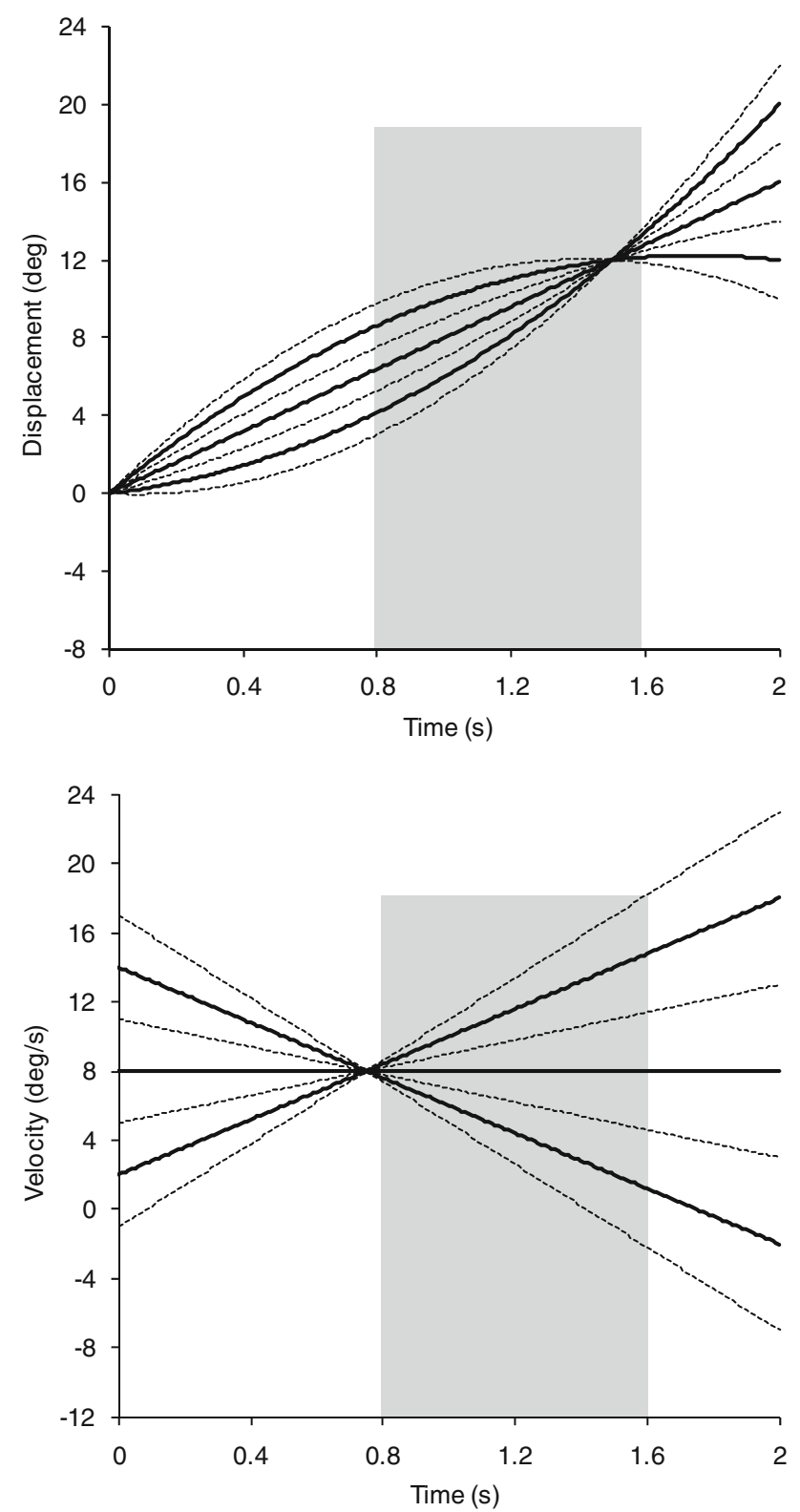

Fig. 1 Representation of pursuit target displacement (upper panel) and velocity (lower panel) versus time (horizontal axis). The pursuit target became visible and moved for $800 \mathrm{~ms}$ with velocity and acceleration characteristics that brought it to pre-occlusion velocity of $8 \mathrm{deg} /$ $\mathrm{s}$ (lower panel). The target was then occluded for $800 \mathrm{~ms}$ (light grey shaded area) and finally reappeared for a further $400 \mathrm{~ms}$. In randomorder trials, target acceleration was $-8,-4,0,4$ or $8 \mathrm{deg} / \mathrm{s}^{2}$, which resulted in five distinct displacement trajectories (upper panel). In blocked-order trials, catch trials (broken line) with target acceleration of $-12,-4,4$, or $12 \mathrm{deg} / \mathrm{s}^{2}$ were interleaved between blocks of test trials (solid line)

before its end. Desaccaded smooth eye velocity was then derived by removing the identified saccades plus 5 additional data points (equivalent to $25 \mathrm{~ms}$ ) at the beginning and end of the identified saccade trajectory from the eye velocity trace (see Fig. 2). The removed data were replaced by a linear interpolation routine based on the smooth eye velocity before and after the saccade. From these data, we extracted from each trial the eye velocity at $50 \mathrm{~ms}$ after the start of the occlusion and $50 \mathrm{~ms}$ after the end of the occlusion; these variables are subsequently referred to as startocclusion and end-occlusion eye velocity. Mean smooth pursuit gain between start-occlusion and end-occlusion was also calculated to give a measure of the overall effectiveness of the smooth response. Total eye displacement during the occlusion (TED) was computed, as was the contribution of the saccadic system (i.e., saccadic eye displacement$\mathrm{SAD})$ in the direction of target motion.

\section{Data analysis}

To determine whether participants were better able to predict accelerative target motion in blocked-order conditions compared to random-order conditions, it was decided to compare measures of pursuit (i.e., velocity and displacement) that were reflective of participants' optimal behaviour in these conditions, and hence minimally influenced by initial inexperience with the paradigm and the target motion characteristics. To this end, we compared the final 6 trials received for each combination of independent variables for targets with $8 \mathrm{deg} / \mathrm{s}$ pre-occlusion velocity; for a detailed analysis of the average ocular response to all combinations of target parameters in the random-order test trials see Bennett et al. (2007). By completing random-order trials before the blocked-order trials, we were able to maximise participants' prior experience and hence expectation regarding the target motion characteristics in the latter stages of the experiment. Accordingly, the intra-participant mean data from the final six trials received for each combination of independent variables were submitted to separate two condition (random-order, blocked-order) $\times 3$ acceleration $(-8$, $\left.0,8 \mathrm{deg} / \mathrm{s}^{2}\right)$ ANOVA. Main and interaction effects were quantified using Tukey's HSD post hoc procedure. Singlesample $T$ tests were also used to determine if group mean measures of pursuit eye movements in test conditions differed from control conditions, as well as corresponding measures of target motion.

A subsidiary analysis examined if there was a difference in the ocular response between the late stages of the random-order conditions and early stages of the blocked-order conditions, and subsequently if there was any adaptation in the ocular response between early and late stages of the blocked-order conditions. Based on findings from previous work showing that participants require only 1-2 repeated trials to scale anticipatory smooth pursuit at the onset of target motion (Barnes et al. 2000; Collins and Barnes 2005), we calculated intra-participant mean data from the first three trials (blocked-order conditions) and the final six trials (blocked-order conditions and random-order conditions) 
Fig. 2 Representative examples from a single participant (P5) of eye position (deg) and velocity (deg/s) vs. time when tracking targets (broken lines) accelerating at $-8 \mathrm{deg} / \mathrm{s}^{2}$ (left panel), $0 \mathrm{deg} / \mathrm{s}^{2}$ (middle panel) and $8 \mathrm{deg} / \mathrm{s}^{2}$ (right panel) received in late random-order (lower panels) or late blocked-order (upper panels) conditions. In the eye position traces, thick parts of the line represent identified saccades. On the eye velocity panels, thick traces represent desaccaded smooth eye velocity, and thin traces indicate saccades. The target initially moves for $800 \mathrm{~ms}$ before being occluded (grey shaded area) for $800 \mathrm{~ms}$ and then reappearing for a further $400 \mathrm{~ms}$
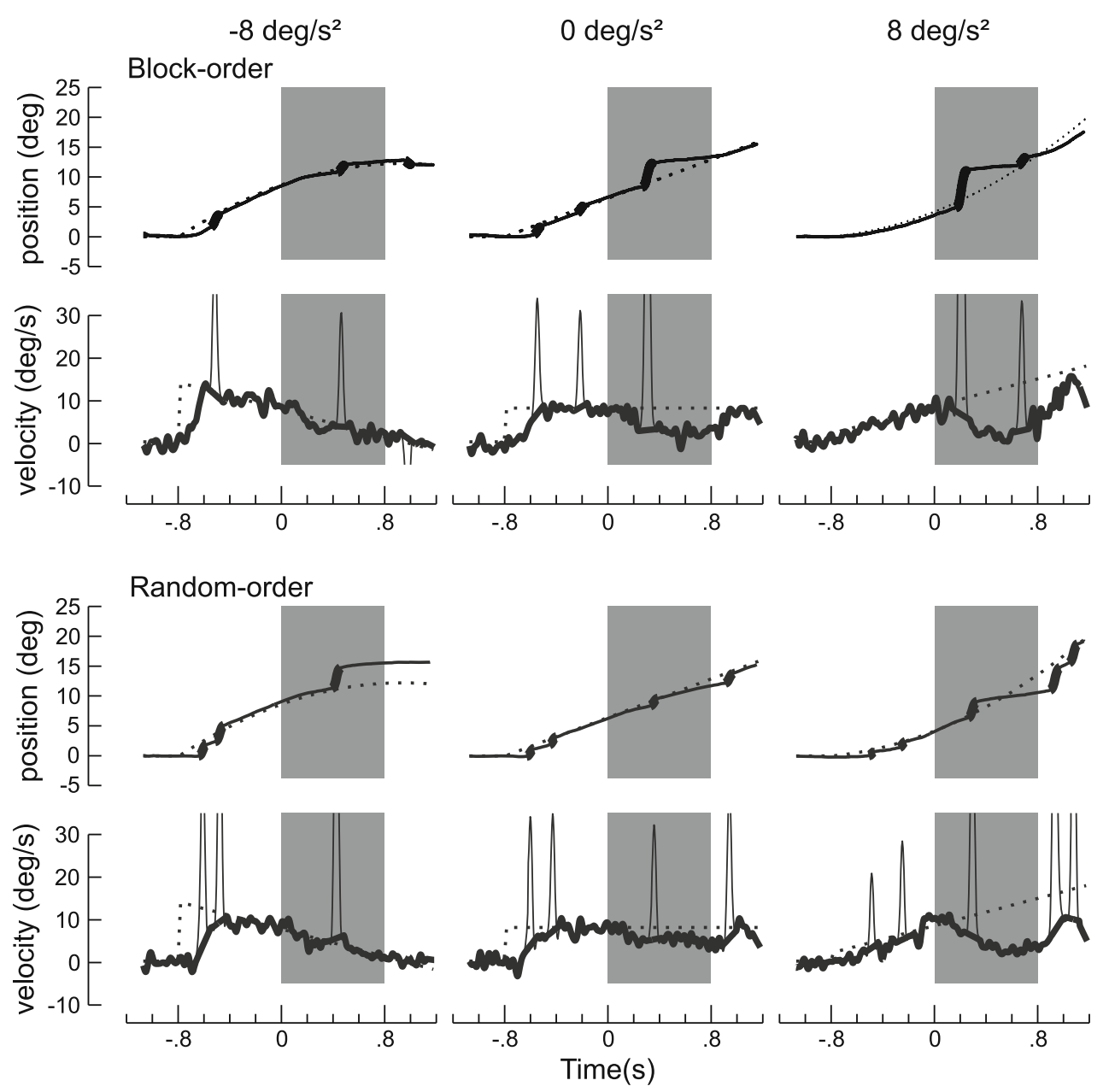

as a measure of the early and late response, respectively. These data were submitted to separate two condition (random late vs. blocked early) or (blocked early vs. blocked late $) \times 3$ acceleration $\left(-8,0,8 \mathrm{deg} / \mathrm{s}^{2}\right)$ ANOVA. Main and interaction effects were quantified using Tukey's HSD post hoc procedure.

Next it was examined whether the oculomotor response during occlusion took account on a trial-by-trial basis of visual feedback available in the initial ramp, or whether it remained scaled to the base target acceleration experienced in the majority of preceding blocked-order trials. To this end, intra-participant mean data from the final six catch trials $\left(-12,-4,4,12 \mathrm{deg} / \mathrm{s}^{2}\right)$ and their immediately preceding blocked-order trials were submitted to three acceleration $\left(-8,0,8 \mathrm{deg} / \mathrm{s}^{2}\right) \times 2$ trial-type (catch, block) ANOVA; catch trials in which acceleration was decreased by $4 \mathrm{deg} / \mathrm{s}^{2}$ were analysed separately from those in which acceleration was increased by $+4 \mathrm{deg} / \mathrm{s}^{2}$. Main and interaction effects were quantified using Tukey's HSD post hoc procedure.

Finally, we sought to determine if there was any evidence of transfer (negative or positive) in catch trials from preceding blocked-order trials. In the event that there were no transfer effects, it should follow that the ocular response in catch trials of a given change in acceleration (e.g. $+4 \mathrm{deg} / \mathrm{s}^{2}$ ) should be equal irrespective of the blocked-order trials in which they were interleaved (i.e., 0 and $+8 \mathrm{deg} / \mathrm{s}^{2}$ ). Furthermore, it would be expected that these catch trials differ by the same amount from random-order trials with equivalent acceleration. To this end, intra-participant mean data were submitted to separate three trial-type (catch $-4 \mathrm{deg} / \mathrm{s}^{2}$, catch $+4 \mathrm{deg} / \mathrm{s}^{2}$, random) $\times 2$ acceleration $(-4$, $+4 \mathrm{deg} / \mathrm{s}^{2}$ ) ANOVA. Main and interaction effects were quantified using Tukey's HSD post hoc procedure. Intraparticipant mean data for random-order trials were calculated from the final 6 trials.

\section{Results}

Pursuit up to and during occlusion

As can be seen in Fig. 2, having pursued the target during the initial visible ramp, eye displacement and velocity at the start of occlusion were well matched to the differing 
levels of target displacement and velocity generated by the three levels of target acceleration (see also Figs. 4, 6). This was confirmed by a significant main effect of acceleration for eye displacement, $F(2,10)=196.8, P<0.001$, and no main effects of acceleration or condition for eye velocity. Start-occlusion eye velocity was equal in the late blockedorder and late random-order trials, and in accordance with the target motion stimulus did not differ as a function of acceleration.

Although the availability of visual feedback during the initial $800 \mathrm{~ms}$ of the trial enabled participants to pursue target motion with good accuracy, smooth eye velocity decayed in test trials when the target was occluded (Fig. 2, upper panel Figs. 3, 4 and 6); no such decay was observed in control trials where visual feedback was available throughout. In trials with $-8 \mathrm{deg} / \mathrm{s}^{2}$ target acceleration, smooth eye velocity tended to decay for the duration of the occlusion. Conversely, in trials with 0 and $8 \mathrm{deg} / \mathrm{s}^{2}$ target acceleration, smooth eye velocity tended to recover back towards target velocity in test trials. As can be seen in Figs. 2, 3 and 4, eye velocity throughout occlusion was somewhat different in the late blocked-order compared to late random-order trials. ANOVA on end-occlusion eye velocity revealed a significant interaction between condition and acceleration, $F(2,10)=12.10, P<0.01$. Post hoc testing indicated that end-occlusion eye velocity showed some scaling in late random-order trials to target acceleration $\left(-8<0\right.$ and $\left.8 \mathrm{deg} / \mathrm{s}^{2}\right)$ but this was more evident in late blocked-order trials and was reflected by differences between each of the three levels of acceleration $\left(-8<0<8 \mathrm{deg} / \mathrm{s}^{2}\right)$. As a consequence of improved scaling, end-occlusion eye velocity was greater in late blockedorder trials than in corresponding late random-order trials when tracking targets with $8 \mathrm{deg} / \mathrm{s}^{2}$ acceleration (upper panel Fig. 3). Still, even though there was evidence of a recovery in smooth eye velocity in anticipation of the end of occlusion, this was not sufficient to match target velocity and eliminate retinal slip. In late random-order trials, group mean end-occlusion eye velocity when tracking targets with 0 and $8 \mathrm{deg} / \mathrm{s}^{2}$ acceleration was less than the corresponding target velocity by -3.6 and $-9.5 \mathrm{deg} / \mathrm{s}$, whereas in late blocked-order trials the difference was -2.1 and $-6.2 \mathrm{deg} / \mathrm{s}$. Single-sample $T$ tests indicated that the undershoot in eye velocity (i.e., the difference between eye velocity and target velocity at the end of occlusion) was significantly different from zero, particularly in late random-order trials $(P<0.01)$.

ANOVA on smooth pursuit gain indicated a main effect of target acceleration, $F(2,10)=14.10, P<0.01$ (middle panel Fig. 3). As can be interpreted from eye velocity throughout occlusion shown in Fig. 4, smooth pursuit gain was considerably less than unity when target acceleration was 0 and $8 \mathrm{deg} / \mathrm{s}^{2}$ and resulted in a significant difference
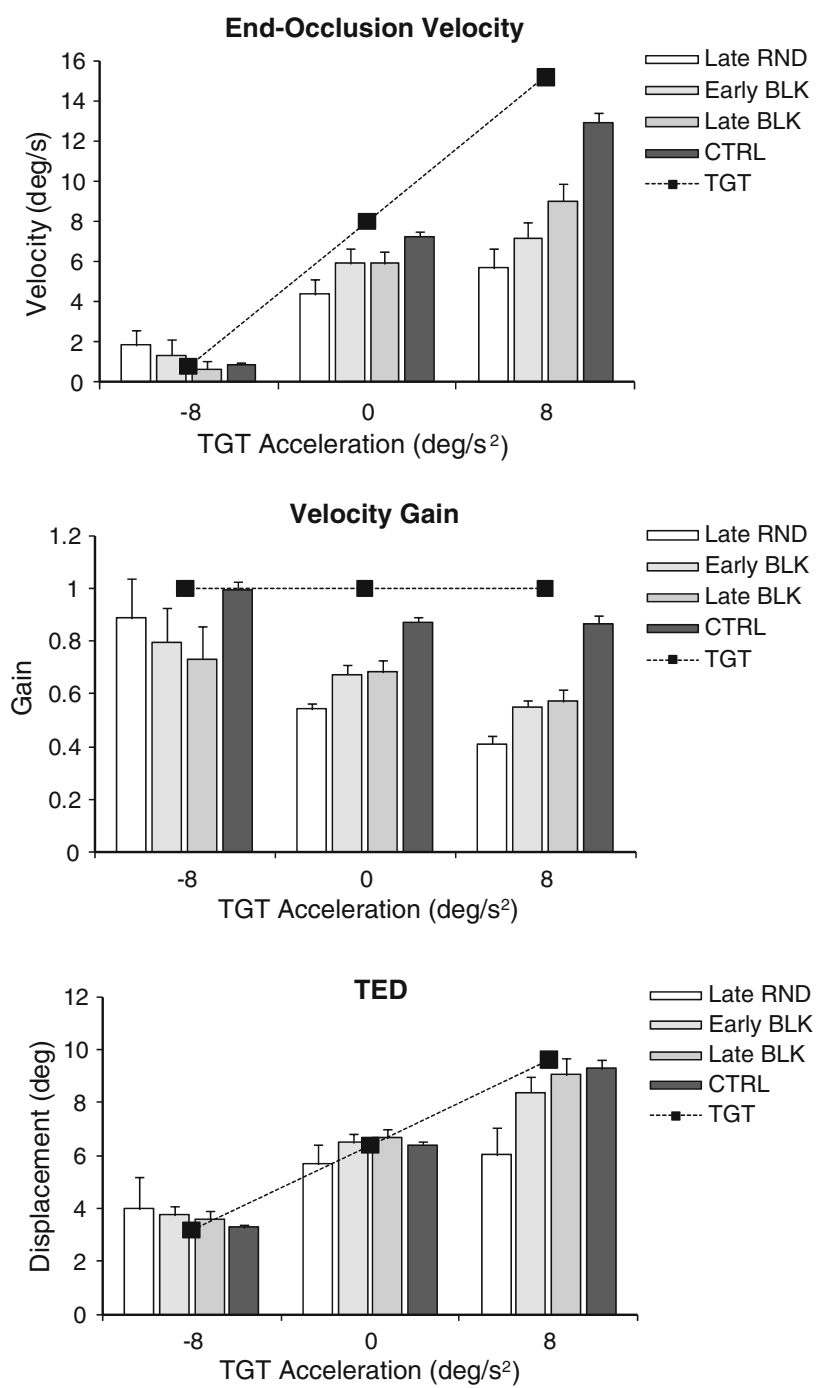

Fig. 3 Group mean (+SE) eye velocity at the end of occlusion (upper panel), smooth pursuit gain (middle panel) and TED (lower panel) as a function of trial order and target acceleration. White bars (late random-order: Late RND), light grey bars (early blocked-order: Early BLK) and dark grey bars (late blocked-order: Late BLK) represent experimental trials, respectively. Black bars represent control trials in which visual feedback was available throughout. Target velocity (diagonal dashed line) is included for comparison

compared to $-8 \mathrm{deg} / \mathrm{s}^{2}$ acceleration. ANOVA did not reveal a significant interaction between condition and acceleration, $F(2,10)=2.86, P>0.05$. However, observation of the group mean data (Fig. 3 middle panel) shows that smooth pursuit gain when tracking targets with 0 and $8 \mathrm{deg} / \mathrm{s}^{2}$ acceleration was generally greater in late blockedorder trials (i.e., 0.68 and 0.57 , respectively) than late random-order trials (i.e., 0.54 and 0.41 , respectively). The reverse pattern was seen when target acceleration was $-8 \mathrm{deg} / \mathrm{s}^{2}$ for the comparison between late blocked-order trials and late random-order trials (i.e., 0.73 and 0.89 , respectively). 

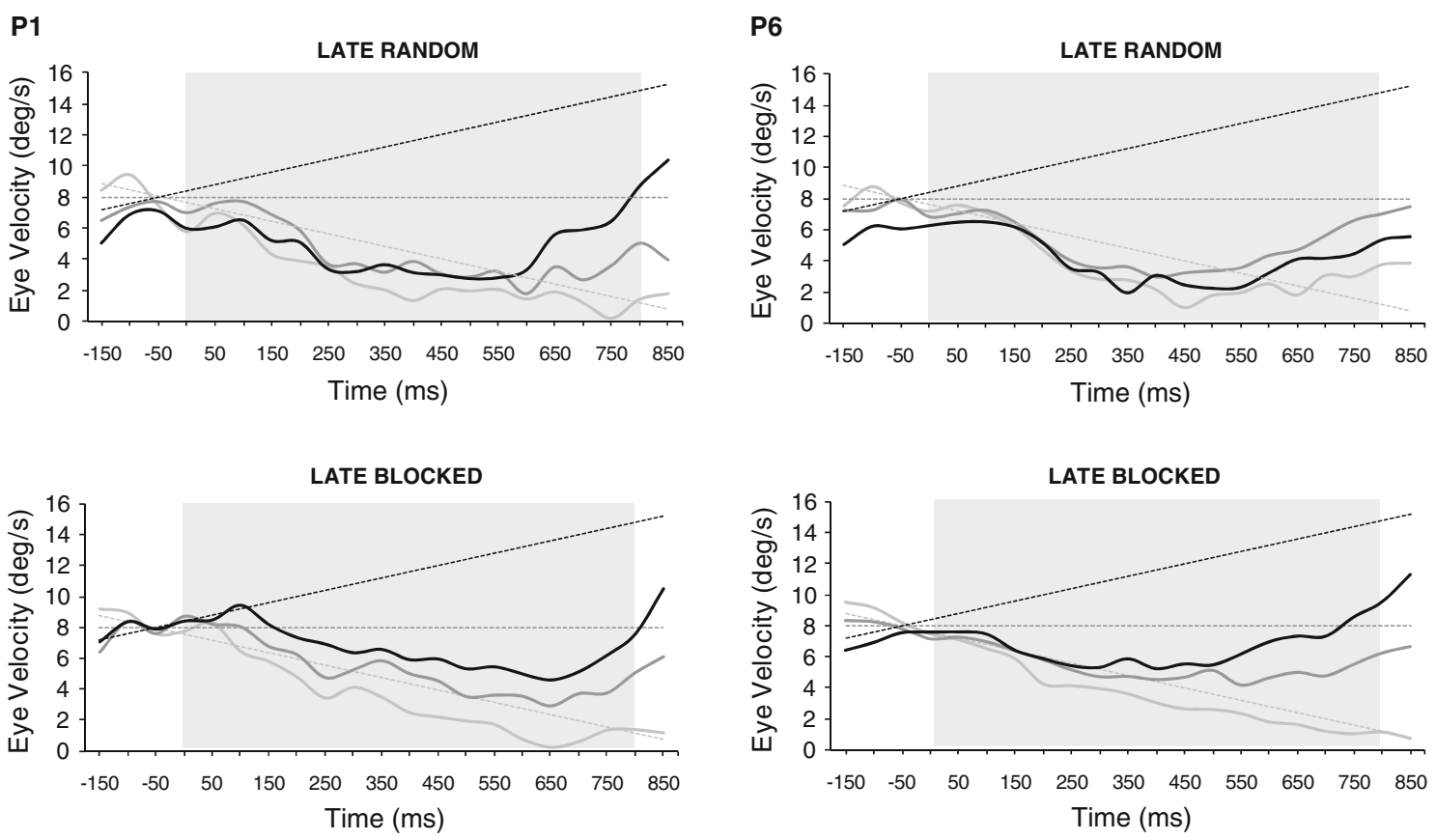

Fig. 4 Representative example from two participants (P1 and P6) of the mean eye velocity vs. time (ms). Eye data (solid lines) are shown when tracking targets (broken lines) accelerating at $-8 \mathrm{deg} / \mathrm{s}^{2}$ (light grey), $0 \mathrm{deg} / \mathrm{s}^{2}$ (dark grey) and $8 \mathrm{deg} / \mathrm{s}^{2}$ (black) received in late

random-order (upper panels) or late blocked-order (lower panels) trials. Target occlusion is represented by the light grey shaded area. Data are shown from $150 \mathrm{~ms}$ before to $50 \mathrm{~ms}$ after target occlusion

\section{Control of eye displacement}

Having shown that end-occlusion eye velocity differed with target acceleration, albeit differentially according to the order in which trials were received (i.e., late blocked vs. late random), we next sought to determine how these measures were reflected in eye displacement during occlusion. Analysis of SAD during occlusion (grey portion of bars in Fig. 5) revealed a main effect of acceleration, $F(2,10)=$ 10.02, $P<0.01$, and a significant interaction between condition and acceleration, $F(2,10)=6.04, P<0.02$. In late blocked-order trials only there was less SAD when tracking targets with -8 and $0 \mathrm{deg} / \mathrm{s}^{2}$ acceleration compared to $8 \mathrm{deg} / \mathrm{s}^{2}$ acceleration. ANOVA on the number of saccades during occlusion revealed no main or interaction effects. Therefore, in conditions when the contribution from SAD was modified, it was achieved by a change in saccade amplitude.

ANOVA on the combined contribution of saccadic and smooth systems (i.e., total eye displacement-TED) revealed a significant main effect of condition, $F(1,5)=$ 13.43, $P<0.02$, and acceleration, $F(2,10)=26.38$, $P<0.01$, as well as a significant condition by acceleration interaction, $F(2,10)=14.21, P<0.01$. As shown in the Fig. 3 (lower panel) and Fig. 5, TED differed between each level of target acceleration in late blocked-order trials, whereas in late random-order trials TED differed only when target acceleration was $-8 \mathrm{deg} / \mathrm{s}^{2}$ compared to 0 or $8 \mathrm{deg} / \mathrm{s}^{2}$

$(P<0.01)$. The improved correspondence between eye and target displacement in late blocked-order trials was confirmed by single-sample $T$ tests, which showed that TED did not differ from target displacement during occlusion for any level of target acceleration $(P>0.05)$. Group mean TED differed from target displacement by between only -0.54 to $0.38 \mathrm{deg}$ (mean $=0.03 \pm 0.5 \mathrm{deg}$ ) in late blocked-order trials. Conversely, in late random-order trials, TED differed from target displacement by between -3.56 and $0.80 \mathrm{deg}$ (mean $=-1.16 \pm 2.21 \mathrm{deg}$ ), which resulted in a significant undershoot when target acceleration was $8 \mathrm{deg} / \mathrm{s}^{2}$.

Adaptation of the oculomotor response

The next analyses determined if there was any change in the ocular response between the late stages of the randomorder conditions and the initial trials of blocked-order conditions (see Fig. 3). ANOVA on intra-participant means from the final six random-order trials and the first three blocked-order trials indicated a significant main effect of acceleration for end-occlusion eye velocity, $F(2,10)=$ 21.29, $P<0.01$. Post hoc testing indicated that in both the late random-order trials and initial blocked-order trials, there was limited evidence of scaling of end-occlusion eye velocity according to target acceleration $\left(-8<0\right.$ and $\left.8 \mathrm{deg} / \mathrm{s}^{2}\right)$. A similar main effect of target acceleration was found for smooth pursuit gain, $F(2,10)=43.63, P<0.01$, which 

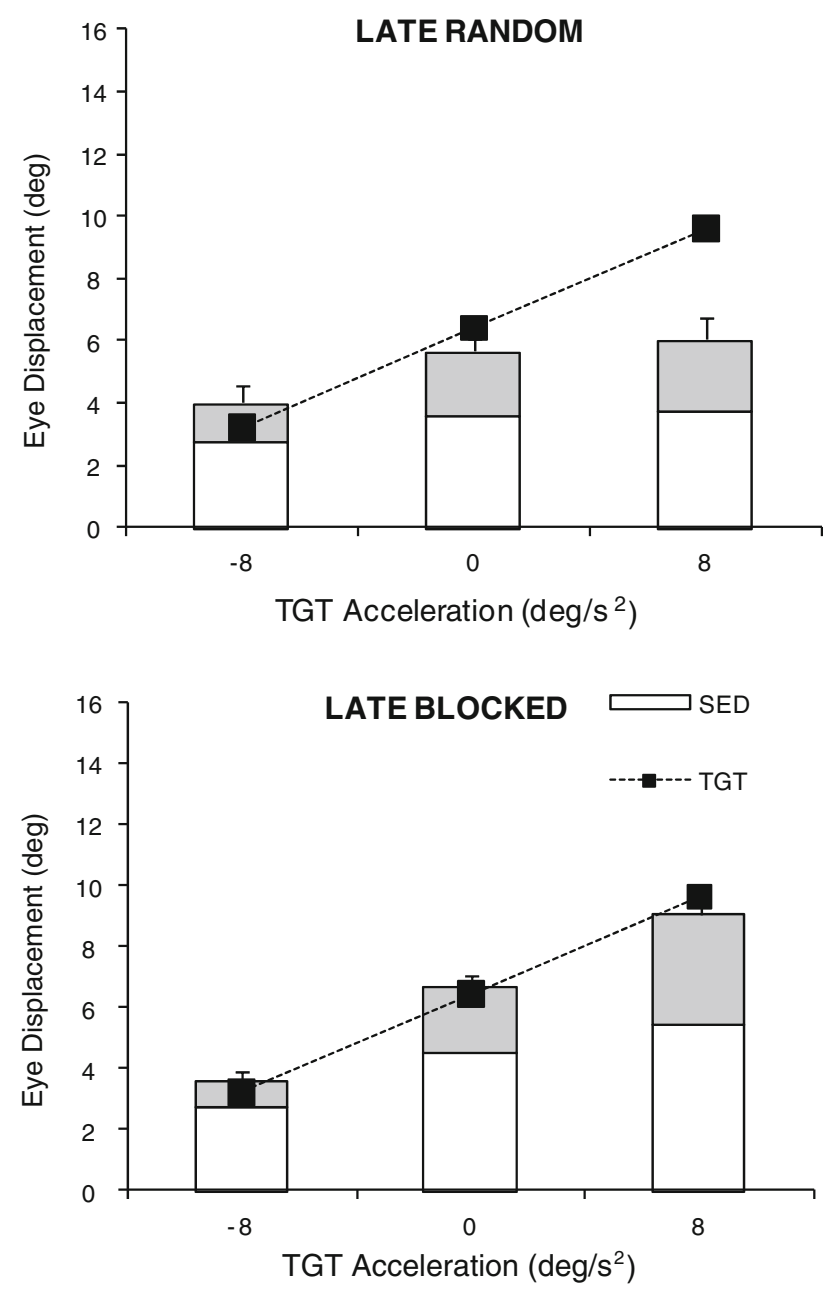

Fig. 5 Group mean (+SE) total eye displacement (TED) during the occlusion interval as a function of trial order (late randomorder $=$ upper panel; late blocked-order $=$ lower panel) and acceleration. Light grey portion of bar represents contribution to displacement from saccades (SAD). Dashed line corresponds to target displacement

became progressively closer to unity as target acceleration was changed from 8 to 0 and then $-8 \mathrm{deg} / \mathrm{s}^{2}$. For TED, there was a significant interaction effect between acceleration and condition, $F(4,20)=5.70, P<0.02$. TED in early blocked-order trials differed according to each level of target acceleration and was greater than in late random-order trials when tracking targets with acceleration of $8 \mathrm{deg} / \mathrm{s}^{2}$ (lower panel Fig. 3).

Subsequently, we examined if there was any adaptation in the ocular response between the early and late stages of the blocked-order conditions. The poor scaling of endocclusion eye velocity to target velocity at reappearance in early blocked-order trials was also present in the comparison between early and late blocked-order trials, which indicated a significant interaction effect, $F(2,10)=4.30$, $P<0.05$ (see Fig. 3 and Fig. 6). Post hoc testing revealed that end-occlusion eye velocity in early blocked-order trials did not differ when target acceleration was 0 or $8 \mathrm{deg} / \mathrm{s}^{2}$. In late blocked-order trials, end-occlusion eye velocity differed between each level of target acceleration $\left(-8<0<8 \mathrm{deg} / \mathrm{s}^{2}\right)$. There was a main effect of acceleration only for TED, $F(2,10)=80.31, P<0.01$ (lower panel Fig. 3). TED differed between each level of target acceleration $\left(-8<0<8 \mathrm{deg} / \mathrm{s}^{2}\right)$. There were no significant main or interaction effects for smooth pursuit gain.

In combination, then, these results suggest that participants were able to quickly modify their response from that exhibited in late random-order trials such that eye displacement was well matched to target displacement. However, three repeated trials in blocked-order with target acceleration of $8 \mathrm{deg} / \mathrm{s}^{2}$ was not sufficient to increase end-occlusion eye velocity above that exhibited in the final six randomorder trials, and hence equal to that achieved in the final six blocked-order trials. Smooth pursuit gain during occlusion was less than unity, particularly when tracking targets with 0 or $8 \mathrm{deg} / \mathrm{s}^{2}$ and did not differ between random-order trials and blocked-order trials, irrespective of the amount of practice in the latter condition. The implication, therefore, is that better scaling of end-occlusion eye velocity in blockedorder trials compared to random-order trials was not linked to higher gain smooth pursuit throughout the occlusion.

\section{Catch trials}

To test whether participants were sensitive to an unexpected change in stimulus characteristics, comparisons were made between catch trials and their immediately preceding blocked-order trials. When acceleration was unexpectedly decreased in catch trials by $4 \mathrm{deg} / \mathrm{s}^{2}$, there was no significant change in end-occlusion eye velocity, $F(1,5)=$ $4.01, P=0.10$, or TED, $F(1,5)=4.96, P=0.10$, compared to preceding blocked-order trials. However, when acceleration in catch trials was increased by $4 \mathrm{deg} / \mathrm{s}^{2}$, there was a significant increase in end-occlusion eye velocity, $(F(1,5)=8.53, P=<0.01)$ and TED, $(F(1,5)=23.30$, $P=0<0.01$ ) (see upper and lower right panels of Fig. 7). Overall, though, it is important to recognise that even when not significantly different the response in catch trials was modulated in the direction expected according to trial-bytrial use of target acceleration.

Having shown that participants did not ignore the visual feedback available from the initial ramp of catch trials, we next sought to determine if there was any evidence of transfer (negative or positive). If there was no transfer at all, it should follow that the ocular response in catch trials of a given acceleration (e.g. $+4 \mathrm{deg} / \mathrm{s}^{2}$ ) should be equal irrespective of the blocked-order trials in which they were interleaved (i.e., 0 and $+8 \mathrm{deg} / \mathrm{s}^{2}$ ). Contrary to this, ANOVA indicated significant interaction effects for both end-occlusion eye velocity, $F(2,10)=34.57, P<0.01$, and TED, 
P4
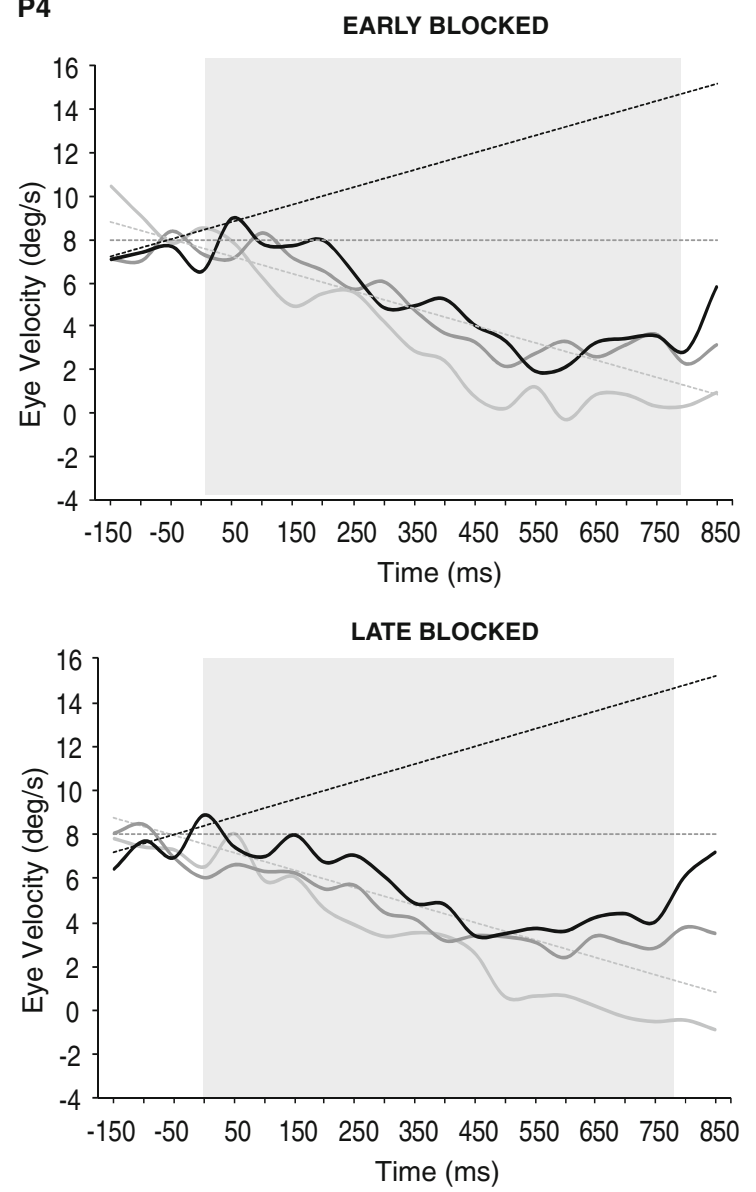

Fig. 6 Representative example from two participants (P3 and P4) of the mean eye velocity vs. time (ms). Eye data (solid lines) are shown when tracking targets (broken lines) accelerating at $-8 \mathrm{deg} / \mathrm{s}^{2}$ (light grey), $0 \mathrm{deg} / \mathrm{s}^{2}$ (dark grey) and $8 \mathrm{deg} / \mathrm{s}^{2}$ (black) received in early

$F(2,10)=22.37, P<0.01$. As the comparison of white bars to grey bars shows in Fig. 8 (left panels), there were significant differences in end-occlusion eye velocity and TED between catch $-4 \mathrm{deg} / \mathrm{s}^{2}$ trials preceded by blockedorder trials of -8 and $0 \mathrm{deg} / \mathrm{s}^{2}$ (end-occlusion velocity: $P=0.001$, TED: $P=0.002$ ). For catch $+4 \mathrm{deg} / \mathrm{s}^{2}$ trials preceded by blocked-order trials of 0 and $8 \mathrm{deg} / \mathrm{s}^{2}$, there was a significant difference in TED $(P=0.04)$; the difference in end-occlusion eye velocity approached conventional levels of significance $(P=0.07)$ (see white versus grey bars in the right panels of Fig. 8).

As a consequence of transfer effects from preceding blocked-order trials, there were also differences between catch trials and the equivalent random-order trials. As can be seen in Fig. 8, these were particularly evident when there was an unexpected decrease in acceleration by $-4 \mathrm{deg} / \mathrm{s}^{2}$. Participants reached higher $(P=0.04)$ endocclusion eye velocity in catch $-4 \mathrm{deg} / \mathrm{s}^{2}$ trials preceded by blocked-order $0 \mathrm{deg} / \mathrm{s}^{2}$ trials (grey bar in upper left panel)
P3
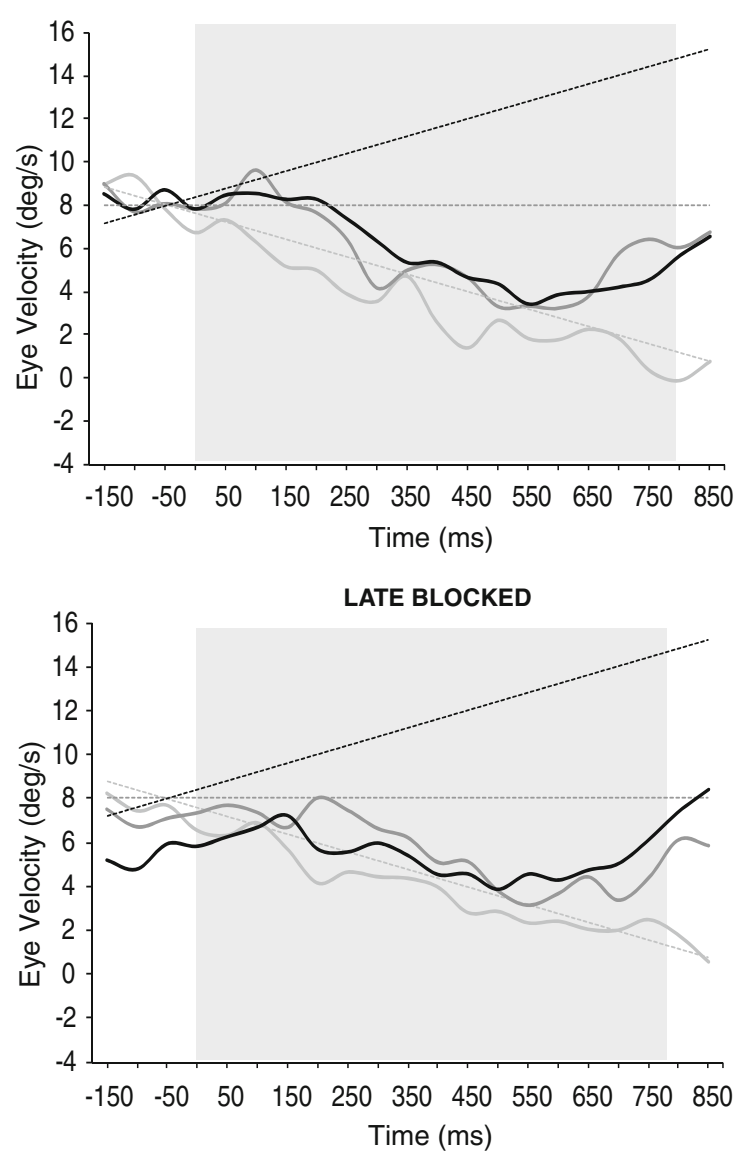

blocked-order (upper panels) or late blocked-order (lower panels) trials. Target occlusion is represented by the light grey shaded area. Data are shown from $150 \mathrm{~ms}$ before to $50 \mathrm{~ms}$ after target occlusion

than in the corresponding random-order trials (black bar in upper left panel). Similarly, end-occlusion eye velocity was higher $(P=0.0002)$ in catch $+4 \mathrm{deg} / \mathrm{s}^{2}$ trials preceded by blocked-order $8 \mathrm{deg} / \mathrm{s}^{2}$ trials (grey bar in upper right panel) than in the corresponding random-order trials (black bar in upper right panel). In addition, TED in these catch trials overshot target displacement and differed from TED in corresponding random-order trials (catch $-4 \mathrm{deg} / \mathrm{s}^{2}$ trials preceded by blocked-order $0 \mathrm{deg} / \mathrm{s}^{2}$ trials: $P=0.003$; catch $+4 \mathrm{deg} / \mathrm{s}^{2}$ trials preceded by blocked-order $8 \mathrm{deg} / \mathrm{s}^{2}$ trials: $P=0.002)$ : compare the grey to black bars in the bottom left and right panels. Following an unexpected increase of acceleration by $+4 \mathrm{deg} / \mathrm{s}^{2}$ in catch trials, there was only limited evidence of modification to end-occlusion eye velocity compared to the equivalent random-order trials (catch $+4 \mathrm{deg} / \mathrm{s}^{2}$ trials preceded by blocked-order $0 \mathrm{deg} / \mathrm{s}^{2}$ trials: $P=0.004)$. There was no significant change to TED and indeed TED was well matched to target displacement in both the random-order and catch trials. 

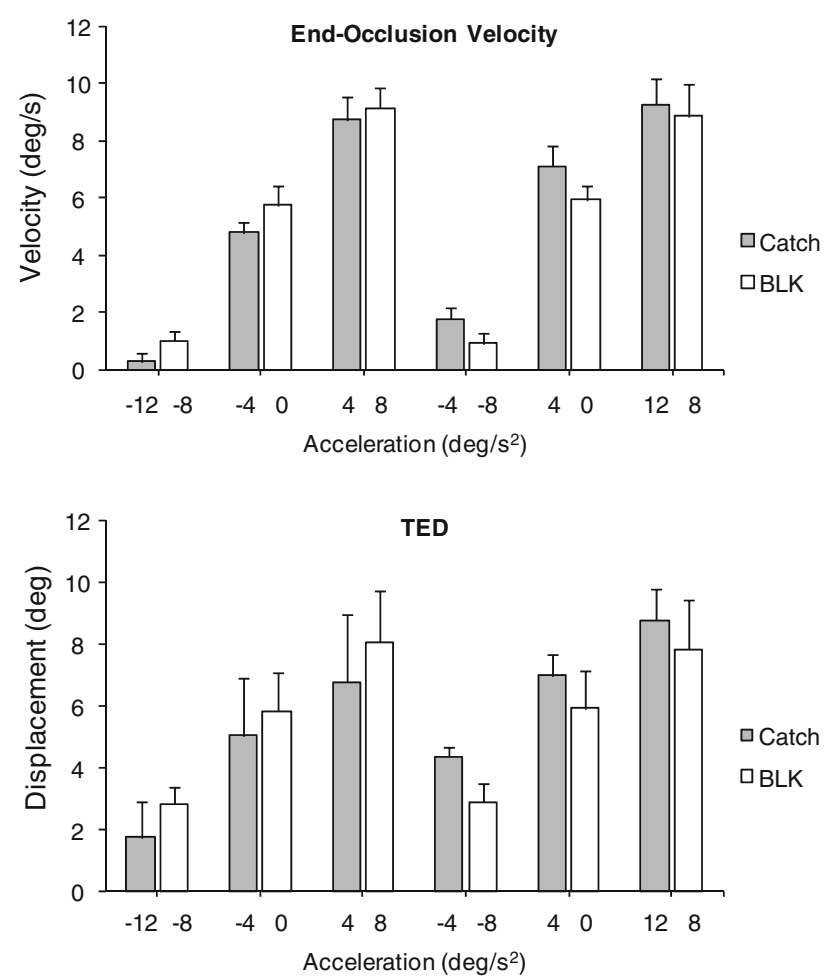

Fig. 7 Group mean (+SE) end-occlusion eye velocity (upper panel) and TED (lower panel) in blocked-order and catch trials as a function of target acceleration. Grey bars represent catch trials white bars represent immediately preceding blocked-order trials

\section{Discussion}

Given that targets moving in our natural surrounds do not often have constant velocity and are frequently occluded from view by other objects, there would be benefit if the oculomotor system formed a prediction that reflected the non-uniform nature of target motion and that could also be retained in the absence of visual feedback. We have shown previously that participants are able to use the visual feedback available prior to an occlusion to scale their ocular response according to accelerative target motion (Bennett et al. 2007). In this case, we hypothesised that participants used an internal dynamic representation of target motion in order to estimate target position and velocity at reappearance and, potentially, throughout the occlusion (see also Orban de Xivry et al. 2008).

It is known that better correspondence during transient occlusion between smooth pursuit eye movements and targets moving with constant velocity develops as participants perform repeated attempts. It is suggested that this is the result of adaptation of the internal drive (Bennett and Barnes 2006b; Madelain and Krauzlis 2003), in combination with increased expectation regarding the target velocity and/or position at reappearance (Becker and Fuchs 1985).
The question remained, therefore, whether repeated attempts to pursue accelerative target motion would similarly enable participants to develop a better prediction of non-uniform occluded target motion and how this would be reflected in the collaboration between smooth and saccadic eye movements during occlusion.

\section{Blocked-order vs. random-order presentation}

Consistent with previous work on human ocular pursuit of constant velocity (Becker and Fuchs 1985; Bennett and Barnes 2003; Orban de Xivry et al. 2006) and accelerating targets (Bennett et al. 2007), we found that having pursued the target well during the initial, visible part of the trial, participants then attempted to maintain pursuit during occlusion using a combination of smooth pursuit eye movements and saccades. Irrespective of the order in which test trials were received, eye velocity initially decayed following target occlusion but then recovered towards levels corresponding to the different target velocities at reappearance. Having completed extensive repeats of the different target motion stimuli, the recovery was better scaled in blockedorder trials. However, despite showing evidence of a recovery in smooth eye velocity in both random-order and blocked-order trials, this was not sufficient to match target velocity at reappearance (see also Bennett and Barnes 2006b) and did not result in near unity smooth pursuit gain. Still, by combining smooth pursuit with a change in saccade amplitude, total eye displacement (TED) during the occlusion in blocked-order trials was well matched to target displacement. The frequency of saccades was similar across the random-order and blocked-order conditions, which is consistent with an earlier occlusion study that showed the predictive saccade trigger was independent of predictive smooth pursuit performance (Orban de Xivry et al. 2009).

An expectation of current models of oculomotor control is that the efficacy of the velocity and positional references would be reduced if the target trajectory were altered on a trial-by-trial basis. The findings from the random-order trials, where participants were relatively poor at matching both eye velocity and displacement to target velocity and displacement are consistent with this prediction. Importantly, these dependent measures of the ocular response were taken after extensive practice had been completed, hence ruling out lack of familiarity with the experimental paradigm and target motion characteristics as a possible explanation. Interestingly, however, randomising trial order did not result in participants using a default smooth response that was reflective of the average target acceleration (i.e., a form of range effect; see Poulton 1975). Such an approach would have done little to minimise undershoot of accelerating targets but would have increased overshoot of decelerating targets. The cost of averaging across the 

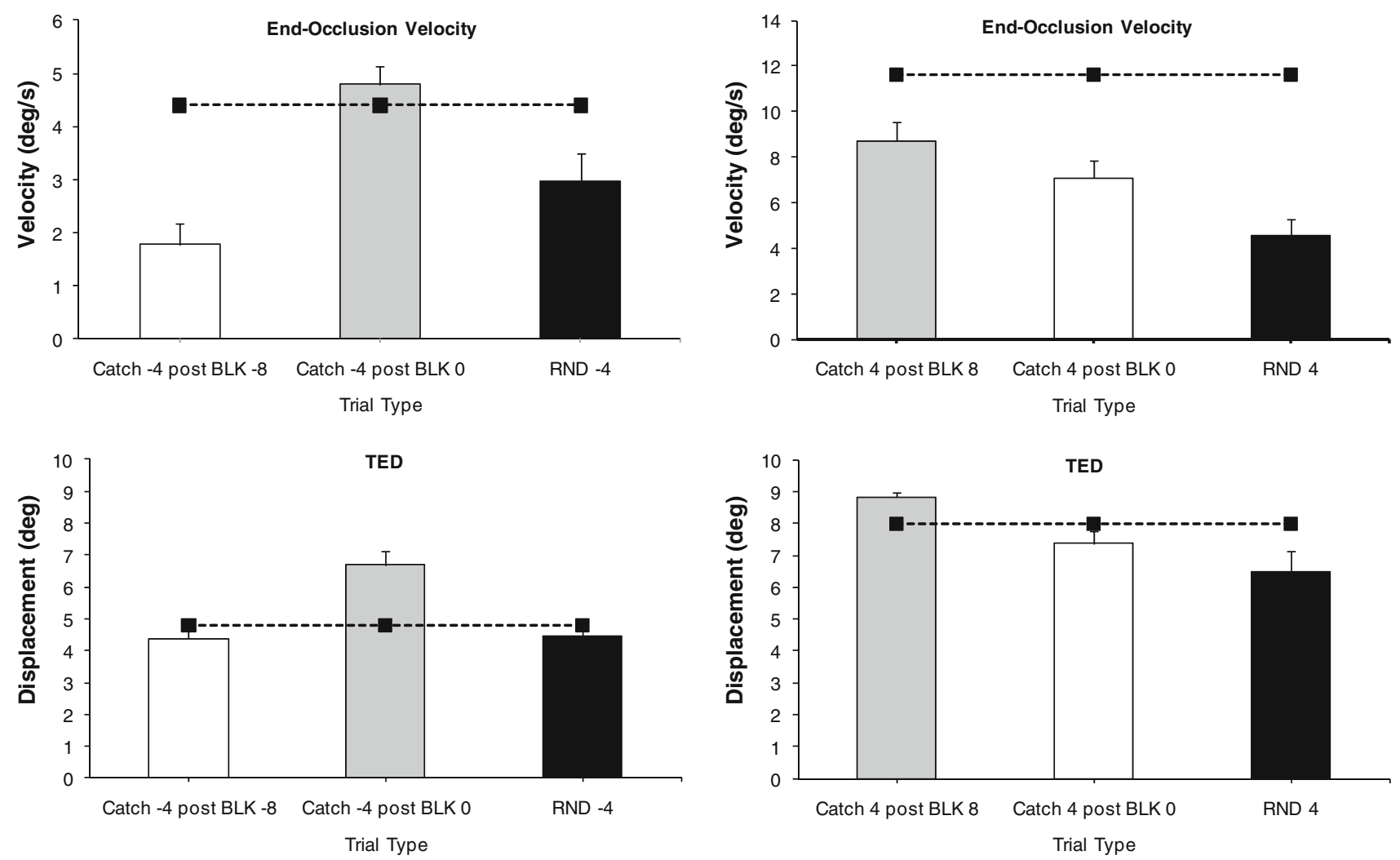

Fig. 8 Group mean (+SE) end-occlusion eye velocity (upper panels) and TED (lower panels) for catch trials and random-order trials of the same acceleration (left panels, $-4 \mathrm{deg} / \mathrm{s}^{2}$; right panels, $+4 \mathrm{deg} / \mathrm{s}^{2}$ ). White bars represent catch trials in which acceleration is increased by

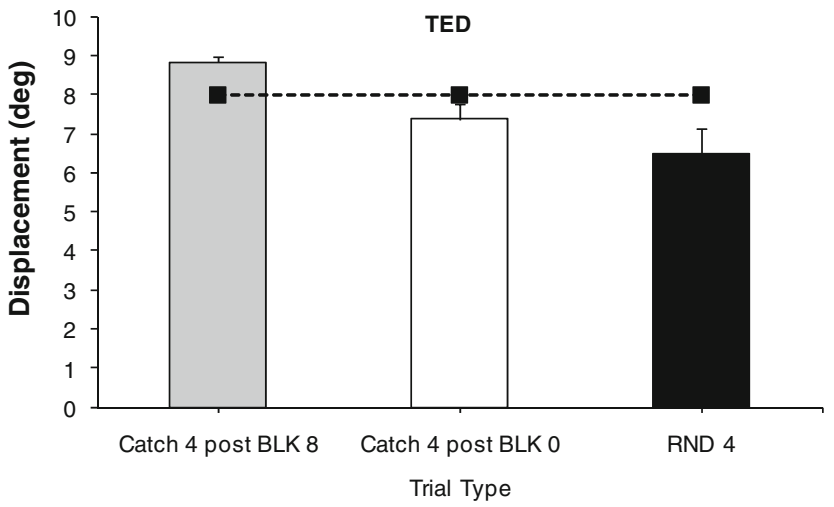

$4 \mathrm{deg} / \mathrm{s}^{2}$; grey bars represent catch trials in which acceleration is decreased by $4 \mathrm{deg} / \mathrm{s}^{2}$. Dashed line corresponds to target velocity and displacement, respectively

different target accelerations would have been particularly evident when considering eye displacement (see Fig. 3 bottom panel and Fig. 5) and would have required reverse saccades to overcome the overshoot of target displacement when tracking targets accelerating at $-8 \mathrm{deg} / \mathrm{s}^{2}$ caused by the smooth response alone. The current data, therefore, are consistent with the suggestion that in the absence of a strong expectation regarding the reappearance position and/ or velocity, participants generate their response on a trialby-trial basis using information available in the initial ramp (Bennett et al. 2007; Orban de Xivry et al. 2006, 2008). Furthermore, it is our contention that although this may not have resulted in a veridical prediction of the future target motion, this behaviour was more advantageous than adopting a default response that did not attempt to take account of the different levels of target acceleration on a trial-bytrial basis.

\section{Adaptation of the oculomotor response}

A subsidiary aim of the current study was to examine if and how the oculomotor response was adapted across the blocked-order trials. In this respect, we found that while participants were quickly able to predict target displacement

of occluded accelerative motion when received in blockedorder (i.e., after three trials), they did not exhibit such good control of eye velocity. End-occlusion eye velocity did not differ between early blocked-order trials and late randomorder trials, and there was limited evidence of scaling in accordance with target acceleration. Better scaling of endocclusion eye velocity was evident after more extensive repeats of the same target motion stimuli received in blocked-order, although this modest adaptation did not enable participants to maintain smooth pursuit gain near unity or match eye velocity to target velocity at the moment the target reappears (see also Madelain and Krauzlis 2003). These results indicate that control of eye position and velocity during a transient occlusion develops at a different rate. However, this interpretation does not contradict with the suggestion that position and velocity representations operate and develop in parallel (see Barborica and Ferrera 2003; Bennett and Barnes 2006a). Instead, it is quite plausible that participants were able to represent the occluded trajectory equally well in terms of position and velocity, but they were less able to exert precise control of smooth pursuit eye movements in the absence of visual feedback. Indeed, it is well known that saccades can be made at will to remembered targets whereas the drive to smooth pursuit 
is more dependent on visual input. Future work is required on this issue.

Predictive oculomotor control is based on memorised and online prediction of target motion

Additional insight on the use of predictive mechanisms in oculomotor control was evident in the comparison of catch trials to blocked-order trials and random-order trials with equivalent acceleration. For instance, we found that participants did not base their response solely on the target motion experienced in the majority of blocked-order trials and simply ignore pre-occlusion information. Instead, there was evidence that eye displacement and eye velocity were modulated according to the target acceleration in these catch trials (see Figs. 7, 8). Therefore, while participants may have had access to a persistent prediction of the upcoming target motion that was used to good effect in blocked-order trials, they still monitored target acceleration and modified their response when there was an unexpected mismatch. Nevertheless, there was also evidence that this trial-by-trial response to the target motion characteristics was influenced by prior blocked-order trials and resulted in cases of positive and negative transfer (for similar effects on smooth pursuit onset see Barnes and Schmid 2002). In this respect, the comparison of catch trials to the preceding blockedorder trials provides novel evidence for the contribution of both long-term (i.e., between-trial) and short-term (i.e., within-trial) predictive mechanisms in ocular pursuit during transient occlusion (Becker and Fuchs 1985).

Acknowledgments This work was supported by the Leverhulme Trust (UK), the Medical Research Council (MRC), the Fonds National de la Recherche Scientifique, the Fondation pour la Recherche Scientifique Médicale, the Belgian Program on Interuniversity Attraction Poles initiated by the Belgian Federal Science Policy Office, internal research grant (Fonds Spéciaux de Recherche) of the Université catholique de Louvain, the European Space Agency (ESA) of the European Union, and the Belgian-American Educational Foundation. The scientific responsibility rests with its authors.

\section{References}

Babler TG, Dannemiller JL (1993) Role of image acceleration in judging landing location of free-falling projectiles. J Exp Psychol Hum Percept Perform 19:15-31

Bahill AT, McDonald JD (1983) Model emulates human smooth pursuit system producing zero-latency target tracking. Bio Cybernet 48:213-222

Barborica A, Ferrera VP (2003) Estimating invisible target speed from neuronal activity in monkey frontal eye field. Nat Neurosci 6:66-74

Barnes GR (2008) Cognitive processes involved in smooth pursuit eye movements. Brain Cogn 68(3):309-326

Barnes GR, Schmid AM (2002) Sequence learning in human ocular smooth pursuit. Exp Brain Res 144:322-335
Barnes GR, Barnes DM, Chakraborti SR (2000) Ocular pursuit responses to repeated, single-cycle sinusoids reveal behaviour compatible with predictive pursuit. J Neurophysiol 84:2340 2355

Becker W, Fuchs AF (1985) Prediction in the oculomotor system: smooth pursuit during transient disappearance of a visual target. Exp Brain Res 57:562-575

Bennett SJ, Barnes GR (2003) Human ocular pursuit during the transient disappearance of a visual target. J Neurophysiol 90:2504-2520

Bennett SJ, Barnes GR (2004) Predictive smooth ocular pursuit during the transient disappearance of a visual target. J Neurophysiol 92:578-590

Bennett SJ, Barnes GR (2006a) Combined smooth and saccadic ocular pursuit during the transient occlusion of a moving visual object. Exp Brain Res 168:313-321

Bennett SJ, Barnes GR (2006b) Smooth ocular pursuit during the transient disappearance of an accelerating visual target: the role of reflexive and voluntary control. Exp Brain Res 175:1-10

Bennett SJ, Orban de Xivry JJ, Barnes GR, Lefevre P (2007) Target acceleration can be extracted and represented within the predictive drive to ocular pursuit. J Neurophys 98:1405-1414

Brouwer AM, Brenner E, Smeets JBJ (2002) Perception of acceleration with short presentation time: can acceleration be used in interception? Percept Psychophys 64:1160-1168

Cerminara N, Apps R, Marple-Horvat DE (2009) An internal model of a moving visual target in the lateral cerebellum. J Physiol 587(2):429-442

Churchland MM, Chou IH, Lisberger SG (2003) Evidence for object permanence in the smooth-pursuit eye movements of monkeys. J Neurophysiol 90(4):2205-2218

Collins CJS, Barnes GR (2005) Scaling of anticipatory smooth eye velocity in response to sequences of discrete target movements in humans. Exp Brain Res 20:1-10

Dallos P, Jones R (1963) Learning behaviour of the eye fixation control system. IEEE Trans Autom Contr AC-8:218-227

de Brouwer S, Missal M, Barnes G, Lefèvre P (2002) Quantitative analysis of catch-up saccades during sustained pursuit. J Neurophysiol 87:1772-1780

Deno DC, Crandall WF, Sherman K, Keller EL (1995) Characterization of prediction in the primate visual smooth pursuit system. BioSys 34:107-128

Leigh RJ, Zee DS (1991) The neurology of eye movements. F.A. Davis Company, Philadelphia

Madelain L, Krauzlis RJ (2003) Pursuit of the ineffable: perceptual and motor reversals during the tracking of apparent motion. J Vision 3:642-653

Nagel M, Sprenger A, Zapf S, Erdmann C, Kömpf D, Heide W, Binkofski F, Lencer R (2006) Parametric modulation of cortical activation during smooth pursuit with and without blanking. An fMRI study. Neuroimage 29(4):1319-1325

Orban de Xivry JJ, Bennett SJ, Lefèvre P, Barnes GR (2006) Evidence for synergy between saccades and smooth pursuit during transient target disappearance. J Neurophysiol 95:418-427

Orban de Xivry JJ, Missal M, Lefèvre P (2008) A dynamic internal representation of target motion drives predictive smooth pursuit during target blanking. J Vision 8(15):1-13

Orban de Xivry JJ, Missal M, Lefèvre P (2009) Smooth pursuit performance during target blanking does not influence the trigger of predictive saccades. J Vision 9(11):1-16

Poulton EC (1975) Range effects in experiments on people. Am J Psych 88(1):3-32

Werkhoven P, Snippe H, Toet A (1992) Visual processing of optic acceleration. Vis Res 32:2313-2329 